\title{
Dimensional stability and dynamic young's modulus of tropical light hardwood chemically treated with methyl methacrylate in combination with Hexamethylene Diisocyanate cross-linker.
}

\begin{abstract}
Chemical treatment is often used to improve the physical and mechanical properties of wood materials. In this study, wood polymer composites (WPC) were prepared from five types of tropical wood species by impregnating the woods with methyl methacrylate (MMA) combined with a cross-linker hexamethylene diisocyanate (HMDIC). Their dimensional stability and mechanical properties were then investigated. The impregnation of wood with monomer systems and polymerization was accomplished through vacuum-pressure and catalyst heat treatment method, respectively. The manufacturing of WPC was confirmed through FT-IR spectroscopy and X-ray diffraction (XRD) analyses. The dimensional stability of manufactured WPC in terms of volumetric swelling (S) and antishrink efficiency (ASE) was measured and found to be improved on treatment. In addition, the modified WPC had lower moisture absorption and higher water repellent efficiency compared to raw wood. The mechanical property of treated samples in terms of dynamics Young's modulus (Ed) was also shown to improve. These improvements in properties were observed as more effective with MMA-HMDIC combination.
\end{abstract}

Keyword: Wood polymer composites (WPC); FT-IR spectroscopy and X-ray diffraction (XRD) analyses. 